\title{
Increase in data capacity utilising dimensions of wavelength, space, time, polarisation and multilevel modulation using a single laser
}

\author{
A. T. Clausen, H. Hu, F. Ye, A. K. Medhin, Y. Ding, R. Asif, M. Galili, T. Morioka, L. K. Oxenløwe \\ DTU Fotonik, Technical University of Denmark, Ørsteds Plads, 2800 Kgs. Lyngby, Denmark \\ Tel: (+45) 4525 3779, e-mail:ancl@fotonik.dtu.dk
}

\begin{abstract}
Increasing the capacity of optical networks while have the objective of lowering the total consumed energy per bit is challenging. By exploiting several dimensions, i.e. wavelength, space, time, polarisation and multilevel modulation simultaneously, a single laser can offer formidable capacity performance with potentially reduced energy consumption per bit. Up to 43 Tbit/s has been demonstrated.
\end{abstract}

Keywords: OTDM, WDM, SDM, multi-core fibre, Nyquist, time-lens

\section{INTRODUCTION}

Data traffic is continuously increasing and predictions indicate that the capacity of individual optical communication systems will soon be too small to carry the network traffic if only frequency/wavelength, time, polarisation and multi-level modulation formats are used [1]. However, by employing a hitherto unused dimension, i.e. space, the capacity may be scaled sufficiently to keep pace [1]. Capacity experiments without Space Division Multiplexing (SDM) demonstrated around 100 Tbit/s [2][3]. With the introduction of multi-core fibres (MCF) the capacity demonstrations were increased significantly showing 305 Tbit/s [4], 1.01 Pbit/s [5] and 1.05 Pbit/s [6] using 19, 12 and 14 cores respectively. In parallel with the quest for more capacity, an additional challenge needs to be addressed, i.e. carbon emission $\left(\mathrm{CO}_{2}\right)$ from the information communications and technology (ICT) industry, which potentially may lead to global warming. ICT $\mathrm{CO}_{2}$ emission contributes with 2 $\%$ of the total emission comparable with avionics and the emission is predicted to double in 2020 [7]. A discussion of energy efficient optical communications can be found in [8]. Thus, both an increase in capacity while lowering the emitted carbon emission per bit, are the challenges. It may be speculated if reducing the number of lasers will reduce the total cost and energy consumption compared to utilise a large number of individual lasers [9]. In this paper 43 Tbit/s using a single laser as seed using wavelength, time, polarisation, quadrature and SDM is presented [10].

\section{CAPACITY INCREASING TECHNIQUES}

Several physical dimensions can be utilised to increase the capacity of optical communication systems, i.e. frequency/wavelength, time, polarisation, quadrature and space. Wavelength and time have been used routinely for years, while polarisation and quadrature have been introduced within the last few years due to cheap fast available Digital Signal Processing (DSP). Finally, space is an untapped commercially deployed resource which may add the extra required capacity needed to keep up with the insatiable capacity need, hence the present focus around the world. The 5 dimensions are briefly summarised below.

Wavelength Division Multiplexing (WDM) has been used for many years and is standardised in the International Telecommunication Union (ITU) standardization body both for coarse and for dense WDM [11][12] and e.g. 370 lasers situated in the $\mathrm{C}$ and L-band with $25 \mathrm{GHz}$ spacing has been reported [2] thus obtaining 101.7 Tbit/s over $3 x 55 \mathrm{~km}$ fibre. Use of many different carriers does not necessarily require multiple separate laser sources. In [13] a single laser source is used to generate 325 sub-carriers.

Using time division multiplexing (TDM) to allocate a number of independent data signals to unique timeslots has been a well-known technique in e.g. SONET/SDH for many years [14]. As the bit rate was increased, the influences of broad spectrum interacting with chromatic dispersion became an increasingly limiting factor combined with the relative low spectral efficiency [15] and thus a direct transition from $10 \mathrm{Gbit} / \mathrm{s}$ to $100 \mathrm{Gbit} / \mathrm{s}$ serial on-off keying (OOK) bit rates will probably not be realised in commercially deployed systems. Besides the expected increased sensitivity to fibre impairments, an additional limitation due to the operation speed of electronics also prevent a smooth transition to even higher serial bit rates. In parallel with the Electrical TDM (ETDM) systems, research groups in the world investigated Optical TDM (OTDM) utilising ultra-narrow temporal pulses emitted from a single laser with repetition rates applicable to electronic bandwidth electronics. By designing a transmitter with parallel branches including modulators and carefully tailored time-delays, the low data-speed optical signals can be multiplexed in time to an ultra-high aggregated bit rate at the output of the transmitter. In 1998 a symbol rate of 640 Gbit/s was demonstrated [16], a record standing more than 10 years 
until 2009 where the symbol rate was doubled to 1.28 Tbit/s [17]. However, by employing quadrature multilevel modulation formats, the bit rate using a single laser was increased further by utilising QAM/QPSK and Polarisation Division Multiplexing (PDM) to 5.1 Tbit/s [18] and 9.5 Tbit/s [19]. As the temporal pulse widths are reduced, the corresponding spectrum is increased simultaneously rendering an efficient use of the available spectrum. In [20] it is suggested and demonstrated how Nyquist pulses in the time domain reduces the spectrum compared to the spectrum from Gaussian or hyperbolic pulses, thus reducing the influence of chromatic dispersion and allowing a more efficient utilisation of the spectrum. The Nyquist pulses are compatible with the OTDM scheme, i.e. N-OTDM [20]. When the bit rate is beyond the bandwidth of electronics, Optical Signal Processing (OPS) is required, e.g. in terms of time-lens schemes briefly addressed in paragraph 3.

With the introduction of Digital Signal Processing (DSP), polarisation rotations and Polarisation Mode Dispersion (PMD) can be tracked and compensated [21]. Thus two orthogonal polarisations can be utilised to carry individual data thus doubling the capacity, i.e. PDM or dual-polarisation [22]. Just as DSP enabled the use of polarisation, DSP also enabled utilising quadrature multi-level modulation formats which spurred a renewed focus as the spectral efficiency is increased compared to OOK modulation [15]. Despite no standardization is available [23] at the line side, $100 \mathrm{Gbit} / \mathrm{s}$ Ethernet is often based on QPSK plus additional PDM [24]. In order to increase the total capacity, the 2 bits per symbol offered by QPSK should in the future be increased. Indeed, impressive high-order level modulation formats have been demonstrated albeit at modest symbol rates; QAM 64 [3], QAM 128 [25], QAM 256 [26], QAM 512 [27], QAM 1024 [28][29].

A hitherto commercially unused dimension is being considered as an additional resource for increasing the data on single fibre [30]. By exploiting space in terms of either multiple cores encapsulated in one single fibre, i.e. MCF, or utilising individual modes as data channels [30] immense capacity increment has been reported as stated in the introduction. The potential capacity of MCF based on number of cores in a fibre and the associated crosstalk is addressed theoretically in [31][32]. Recently a MCF with 36 cores has been reported emphasising the potential for increasing the capacity significantly [33]. For recent comprehensive surveys of MMF please refer to [30][34][35]. Coupling in and out of MCFs require special attention to the fan-in fan-out (FI/FO) devices [34]. In [36] an on-chip 7-core FI/FO device on silicon-on-insulator platform is reported and utillised in [37] to transmit 1.2 Tbaud/s DPSK in each of the 6 cores and a clock signal in the $7^{\text {th }}$ core. In parallel with multiple cores in the fibre, using photonic lanterns different modes can be specifically exited and carry independent data [30]. In [38] 15 spatial modes are supported and transmitted over $23.8 \mathrm{~km}$ of fibre.

\section{EXPERIMENTAL SET-UP AND RESULTS}

In Figure 1 the experimental set-up for generating and evaluating 43 Tbit/s using a single laser as seed is depicted [10]. An erbium glass oscillating mode-locked laser (ERGO-MLL) with a repetition frequency of 10 $\mathrm{GHz}$ is used. The interplay between high peak power and non-linearity in a dispersion flattened highly nonlinear fibre (DF-HNLF) generates a broad self-phase modulation (SPM) induced spectrum, which is used to carve out a clock signal and a signal part. The signal is QPSK modulated before being multiplexed to 320 Gbit/s using passive delay lines and finally polarisation multiplexed. In total, a 1.28 Tbit/s, i.e. 1.2 Tbit/s after subtracting 7 $\%$ FEC overhead, is generated at the output of the polarisation multiplexer. A Wavelength Selective Switch (WSS) is used to emulate a 6 wavelength WDM signal, each outlet of the WSS additionally induce a rectangular filtering at $320 \mathrm{GHz}$, thus generating a 320 Gbaud N-OTDM signal at each WDM wavelength. Using delay lines, splitters and amplifiers, 6 de-correlated SDM channels are generated and launched into the 6 outer cores of the MCF, while the clock signal is launched into the centre core, using a free-space (FI/FO) device.

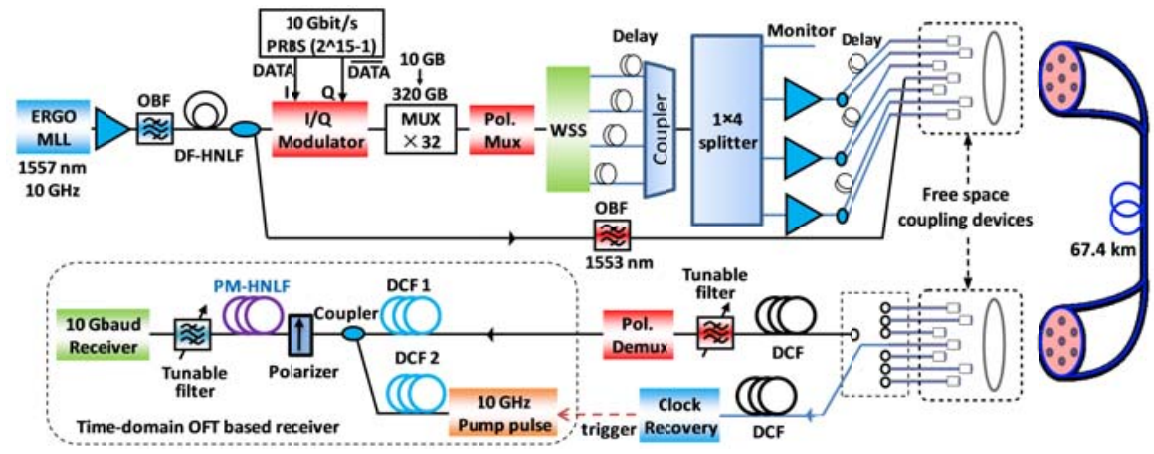

Figure 1.Experimental set-up used for generating and detecting 43 Tbit/s[10].

At the receiver, the clock signal after dispersion compensation is launched into a clock recovery circuit, which is used to generate the required trigger signal needed for the local laser source constituting the pump in a time-lens 
scheme [39]. The data signal is dispersion compensated and polarisation demultiplexed before launched into the time-lens and converted from 320 Gbaud N-OTDM to an Optical Frequency Division Multiplexed (OFDM) signal with a sufficient large channel spacing to allow filtering of each individual channel using a $40 \mathrm{GHz}$ tuneable optical bandpass filter. In Figure 2 the BER characterisation of all the total 2304 tributaries channels (SDM +WDM+OTDM in both polarisations) is shown. The BER is below BER $=3.8 \cdot 10^{-3}$ corresponding to the FEC threshold. As the I\&Q components showed identical performance, it was concluded that successful transmission of all channels were obtained.

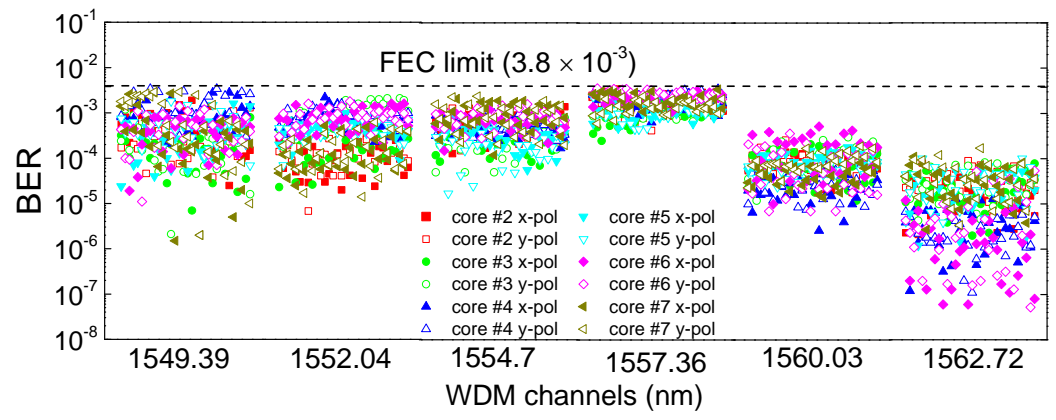

Figure 2. BER of all 2304 tributaries [10].

\section{CONCLUSIONS}

In this paper a single laser used as the seed to generate 43 Tbit/s deploying WDM, OTDM, PDM, SDM and QPSK is presented, motivated and discussed. The experimental characterisation shows error-free operation below the FEC threshold for all channels.

\section{REFERENCES}

[1] R-J. Essiambre, et al.: Capacity Trends and Limits of Optical Communication Networks, Proc. IEEE, Vol. 100, No. 5, pp. 1035-1055 (2012).

[2] D. Qian, et al.: 101.7-Tb/s (370x294-Gb/s) PDM-128QAM-OFDM Transmission over 3x55 km SSMF using Pilot-based Phase Noise Mitigation”, Proc. OFC 2011, Los Angeles, California, USA, 2011, paper PDPB5 (2011).

[3] A. Sano, et al.: 102.3-Tb/s (224x548-Gb/s) C- and Extended L-band All-Raman Transmission over 240 km Using PDM-64QAM Single Carrier FDM with Digital Pilot Tone, Proc. OFC 2012, Los Angeles, California, USA, paper PDP5C.3 (2012).

[4] J. Sakaguchi, et al.: 19-core fiber transmission of 19x100x172-Gb/s SDM-WDM-PDM-QPSK signals at 305Tb/s, Proc. OFC 2012, Los Angeles, California, USA, paper PDP5C.1 (2012).

[5] H. Takara, et al.: 1.01-Pb/s (12 SDM/222 WDM/456 Gb/s) Crosstalk-managed Transmission with 91.4b/s/Hz Aggregate Spectral Efficiency”, Proc. ECOC 2012, Amsterdam, Netherland, Post deadline paper Th.3.C.1 (2012).

[6] D. Qian, et al.: 1.05Pb/s Transmission with 109b/s/Hz Spectral Efficiency using Hybrid Single- and FewMode Cores, Proc. FIO 2012, Rochester, New York, USA, paper FW6C.3 (2012).

[7] C. A. Chan, et al.: Methodologies for Assessing the Use-Phase Power Consumption and Greenhouse Gas Emissions of Telecommunications Network Services, Environmental Science \& Technology, Vol. 47, No. 1, pp 485-492 (2013).

[8] D. C. Kilper, et al.: Energy-efficient Telecommunications, Optical Fiber Telecommunications VIB Systems and Networks, Chapter 17, Elsevier (2013).

[9] D. Hillerkuss, et al.: Single-Laser 32.5 Tbit/s Nyquist WDM Transmission, J. Opt. Commun. Netw., Vol. 4, No. 10, pp. 715-723 (2012).

[10] H. Hu, et al.: Single Source 5-dimensional (Space-, Wavelength- , Time- ,Polarization-, Quadrature-) 43 Tbit/s Data Transmission of $6 \mathrm{SDM} \times 6 \mathrm{WDM} \times 1.2 \mathrm{Tbit} / \mathrm{s}$ Nyquist-OTDM-PDM-QPSK, Proc. CLEO 2014, San Jose, California, USA , paper JTh5B (2014)

[11] ITU-T G.694.2 (12/2003), Telecommunication standardization sector of ITU. Series G: Transmission systems and media, digital systems and networks. Transmission media characteristics - Characteristics of optical components and subsystems. Spectral grids for WDM applications: CWDM frequency grid.

[12] ITU-T G.694.1 (12/2003), Telecommunication standardization sector of ITU. Series G: Transmission systems and media, digital systems and networks. Transmission media characteristics - Characteristics of optical components and subsystems. Spectral grids for WDM applications: DWDM frequency grid.

[13] D. Hillerkuss, et al.: 26 Tbit $\mathrm{s}^{-1}$ line-rate super-channel transmission utilizing all-optical fast Fourier transform processing, Nature Photonics, Vol 5, pp 364-371 (2011). 
[14] E. Lach, et al.: Challenges for 100 Gbit/s ETDM Transmission and Implementation, Proc. OFC 2007, Anaheim, California, USA, paper OWE1 (2007).

[15] E. Lach, et al.: Modulation formats for 100G and beyond, Optical Fiber Technology, Vol. 17, pp. 377-386 (2011).

[16] M. Nakazawa, et al.: TDM single channel 640 Gbit/s transmission experiment over $60 \mathrm{~km}$ using $400 \mathrm{fs}$ pulse train and walk-off free, dispersion flattened nonlinear optical loop mirror, Electronics Letters, Vol. 34, No. 9, pp. 907-908 (1998).

[17] H. C. Hansen Mulvad, et al.: 1.28 Tbit/s single-polarisation serial OOK optical data generation and demultiplexing, Electronics Letters, Vol. 45, No. 5, pp. 280-281 (2009).

[18] H. C. Hansen Mulvad, et al.: Demonstration of 5.1 Tbit/s data capacity on a single-wavelength channel, Optics Express,Vol. 18, No. 2, pp. 1438-1443 (2010).

[19] T. Richter, et al.: Single Wavelength Channel 10.2 Tb/s TDM-Data Capacity using 16-QAM and Coherent Detection, Proc. OFC 2011, Los Angeles, California, USA, 2011, paper PDPA9.

[20] M. Nakazawa et al.: Ultrahigh-speed “orthogonal” TDM transmission with an optical Nyquist pulse train, Optics Express, Vol. 20, No. 2, pp. 1129-1140 (2012).

[21] E. M. Ip, et al.: Fiber Impairment Compensation Using Coherent Detection and Digital Signal Processing, Journal of Lightwave Technology, Vol. 28, No. 4, pp. 502 - 519 (2010).

[22] Infinera: White paper Coherent DWDM Technologies, Document number WP-CT-10-2012 (2012).

[23] JDSU Guylain Barlow: White paper Testing 100 G Transport Networks and Services (2013).

[24] Optical Internetworking Forum (OIF): Implementation Agreement for Integrated Dual Polarization Intradyne Coherent Receivers, Document number IA \# OIF-DPC-RX-01.0 (2010).

[25] D. Qian, et al.: 101.7-Tb/s (370x294-Gb/s) PDM-128QAM-OFDM Transmission over 3x55 km SSMF using Pilot-based Phase Noise Mitigation, Proc. OFC 2011, Los Angeles, California, USA, 2011, paper PDPB5.

[26] T. Omiya, et al. :400 Gbit/s 256 QAM-OFDM transmission over $720 \mathrm{~km}$ with a $14 \mathrm{bit} / \mathrm{s} / \mathrm{Hz}$ spectral efficiency by using high-resolution FDE, Optics Express, Vol. 21, No. 3 , pp. 2632-2641 (2013).

[27] Y. Koizumi, et al. : 512 QAM transmission over $240 \mathrm{~km}$ using frequency-domain equalization in a digital coherent receiver, Optics Express, Vol. 20, No. 21, pp. 23383-23389 (2012).

[28] M-F. Huang, et al.:50.53-Gb/s PDM-1024QAM-OFDM Transmission using Pilot-based Phase Noise Mitigation, Proc. OECC 2011, Kaohsiung, Taiwan, pp. 752 - 753 (2011)

[29] Y. Koizumi, et al.: 1024 QAM (60 Gbit/s) single-carrier coherent optical transmission over $150 \mathrm{~km}$, Optics Express, Vol. 20, No. 11, pp. 12508-12514 (2012)

[30] D. J. Richardson, et al.: Space Division Multiplexing in Optical Fibres, Nature Photonics, Vol. 7, pp. 354362 (2013).

[31] F. Ye, et al.: Capacity of Space-Division Multiplexing with Heterogeneous Multi-Core Fibers, Proc. OECC 2013, Kyoto, Japan, paper WR2-3.

[32] F. Ye, et al.: High-count Multi-Core Fibers for Space-Division Multiplexing with Propagation-Direction Interleaving, Proc. OFC 2015, California, USA, paper Th4C.3 (2015).

[33] J. Sakaguchi, et al.: Realizing a 36-core, 3-mode Fiber with 108 Spatial Channels, Proc. OFC 2015, California, USA, paper Th5C.2 (2015).

[34] K. Saitoh, et al.: Multicore fibers for large capacity transmission, De Gruyter, Nanophotonics, Vol. 2, No. 5-6, pp. 441-454 (2013)

[35] T. Hayashi, et al.: Multi-core Fiber for High-Capacity Long-Haul Spatially-Multiplexed Transmission, SEI Technical review, No. 77, pp. 14-22 (2013).

[36] Y. Ding, et al.: On-chip Grating Coupler Array on the SOI Platform for Fan-in/Fan-out of Multi-core Fibers with Low Insertion Loss and Crosstalk, Proc. ECOC 2014, Cannes, France, paper We.1.1.3.

[37] H. Hu, et al.: 1.28 Tbaud Nyquist-OTDM Transmission over a 7-Core Fiber Using an On-Chip SDM Coupler, Proc. ECOC 2014, Cannes, France, paper THPDP2-2.

[38] N. K. Fontaine, et al.: 30x30 MIMO Transmission over 15 Spatial Modes, Proc. OFC 2015, California, USA, paper Th5C.1.

[39] A. T. Clausen, et al.: All-optical signal processing of OTDM and OFDM signals based on Time-domain Optical Fourier Transformation, Proc. ICTON 2014, Graz, Austria, paper We.A1.5 (2014). 